Protocols

\title{
Electrospun ultrathin PBAT/nHAp fibers influenced the in vitro and in vivo osteogenesis and improved the mechanical properties of neoformed bone
}

\author{
Gabriela F. Santana-Melo ${ }^{\mathrm{a}, 1}$, Bruno V.M. Rodrigues ${ }^{\mathrm{b}, 1}$, Edmundo da Silva ${ }^{\mathrm{c}}$, \\ Ritchelli Riccic ${ }^{\mathrm{C}}$, Fernanda R. Marciano ${ }^{\mathrm{b}, \mathrm{c}, \mathrm{d}, \mathrm{e}}$, Thomas J. Webster ${ }^{\mathrm{e}}$, \\ Luana M.R. Vasconcellos ${ }^{\mathrm{a}, * *}$, Anderson O. Lobo ${ }^{\mathrm{b}, \mathrm{c}, \mathrm{d}, \mathrm{e}, *}$ \\ ${ }^{a}$ Department of Bioscience and Oral Diagnosis, Institute of Science and Technology, Sao Paulo State University, Sao Jose dos Campos, Sao Paulo, Brazil \\ ${ }^{\mathrm{b}}$ Laboratory of Biomedical Nanotechnology, Universidade Brasil, Itaquera, Sao Paulo, Brazil \\ ${ }^{\mathrm{c}}$ Laboratory of Biomedical Nanotechnology, Institute of Research and Development (IPED), University of Vale do Paraiba, Sao Jose dos Campos, Sao Paulo, \\ Brazil \\ d Biomaterials Innovation Research Center, Department of Medicine, Brigham and Women's Hospital, Harvard Medical School, Cambridge, USA \\ e Nanomedicine Laboratory, Department of Chemical Engineering, Northeastern University, Boston, MA, USA
}

\section{A R T I C L E I N F O}

\section{Article history:}

Received 16 October 2016

Received in revised form 30 March 2017

Accepted 24 April 2017

Available online 27 April 2017

\section{Keywords:}

Electrospinning

PBAT

Nanohydroxyapatite

Gene expression

Micro-computed tomography

Bone regeneration

\begin{abstract}
A B S T R A C T
Combining polyester scaffolds with synthetic nanohydroxyapatite (nHAp), which is bioactive and osteoconductive, is a plausible strategy to improve bone regeneration. Here, we propose the combination of PBAT [poly(butylene-adipate-co-terephthalate)] and synthetic nHAp (at 3 and 5 wt\%). PBAT is a relatively a new polymer with low crystallinity and attractive biodegradability and mechanical properties for orthopedic applications, however, with a still underexplored potential for in vivo applications. Then, we performed a careful biological in vitro and in vivo set of experiments to evaluate the influence of PBAT containing two different nHAp loads. For in vitro assays, osteoblast-like MG63 cells were used and the bioactivity and gene expression related to osteogenesis were evaluated by qRT-PCR. For in vivo experiments, twenty-four male rats were used and a tibial defect model was applied to insert the scaffolds. Micro-computed tomography (Micro-CT) and histological analysis were used to assess e bone neoformation after 6 weeks of implantation. Three point flexural tests measured the mechanical properties of the neoformed bone. All scaffolds showed promising in vitro properties, since they were not cytotoxic against MG-63 cells and promoted high cell proliferation and formation of mineralized nodules. From a mechanistic point-of-view, nHAp loading increased hydrophilicity, which in turn allowed for a better adsorption of proteins and consequent changes in the phenotypic expression of osteoblasts. nHAp induced better cellular responses on/in the scaffolds, which was mainly attributed to its osteoconductive and osteoinductive properties. Micro-CT images showed that nHAp at $3 \%$ and 5 wt $\%$ led to more effective bone formation, presenting the highest bone volume after 6 weeks of implantation. Considering the three point flexural tests, $5 \mathrm{wt} \%$ of nHAp positively influenced the flexural mode of the neoformed bone, but the stiffiness was similar between the 3\% and 5 wt\% groups. In summary, this investigation demonstrated great potential for the application of these novel scaffolds towards bone regeneration and, thus, should be further studied.
\end{abstract}

(c) 2017 Elsevier B.V. All rights reserved.

\footnotetext{
* Corresponding author at: Laboratory of Biomedical Nanotechnology, Universidade Brasil, Itaquera, Sao Paulo, Brazil.

** Corresponding author.

E-mail addresses: luana@ict.unesp.br (L.M.R. Vasconcellos), aolobo@mit.edu, lobo.aol@gmail.com, aolobo@cnpq.br(A.O. Lobo).

1 These authors contributed equally to this work.
}

\section{Introduction}

In recent decades, the development of natural and synthetic biomaterials has aroused the interest of researchers around the world in order to induce and guide fast and controlled tissue regeneration seeking to improve the quality of life. The reconstruction of bone defects, such as local filling of lost bone tissue due to trauma, infectious processes or congenital malformations, is an ordinary procedure in maxilo-facial and orthopedic surgeries, however, it 
represents a major challenge to medical and dental clinics due to a lack of prolonged bone growth, infection, and other reasons [1-3].

Several techniques have been investigated for the preparation of improved orthopedic biomaterials, many focused on developing biologicially-inspired nanometer surfaces. Particulatly, electrospinning has been studied as an alternative to produce 3D fibrous scaffolds due to an ability to mimic the architecture of the bone extracellular matrix (ECM) [4-6]. Electrospinning presents attractive features, such as the production of uniform and continuous ultrathin fibers ( $100<$ diameters $<1000 \mathrm{~nm}$ ) and nanofibers (diameters $<100 \mathrm{~nm}$ ), which in turn possess an elevated surface area, high porosity, interconnectivity and the possibility of large-scale production [6-8]. Additionally, electrospinning is considered to be a simple approach of low-cost and high versatility, since the same system may be used for processing both natural and synthetic materials, along with the easy incorporation of functional components, such as proteins, growth factors, genes, enzymes, drugs, bioactive ceramics and others, making the final materials ideal candidates for numerous tissue engineering applications $[5,6,9,10]$.

Such electrospun ultrathin and nanometer fibers can be obtained using a great variety of natural or synthetic polymers as matrices. In order to produce novel scaffolds, biodegradable polymers have been extensively investigated [10]. Among them, synthetic aliphatic polyesters stand out due to their controllable biodegradability (PLA [11,12], PGA [13,14], PLGA [15,16], and PCL[13,17]). However, these polyesters are usually rigid and highly crystalline, which may impair some in vivo interactions. Meanwhile, PBAT (poly (butylene adipate-co-terephthalate) is an interesting polyester due to a high flexibility and elongation-atbreak besides low crystallinity $[18,19]$. Due to these properties, PBAT has an adjustable balance between optimal biodegradation and desirable physical-chemical-properties [18,20,21]. Besides poor mechanical properties and relatively low bioactivity, PBAT still presents an underexplored potential for in vivo applications. In order to solve these drawbacks, some have incorporated specific nanoparticles into PBAT polymeric matrices for simultaneously enhancing mechanical and biofunctionality properties of the final material [22-24].

The synthesized nanohydroxyapatite (nHAp) is similar to that found in bone in terms of chemical structure and spatial organization $[19,25,26]$. The nHAp exhibits excellent biocompatibility, bioactivity and osteoconductivity $[27,28]$. Due to these properties, nHAp has been one of the most highly investigated materials for the replacement and regeneration of bone tissues [29-31].

It is known that the fine control of surface wettability is key to obtaining a deep understanding of the processes of biological cell integration and the further development of new potential biomaterials for the tissue engineering community [32]. The wettability of biomaterials is a crucial factor for promoting select protein interactions (adsorption as well as bioactivity) and consequently adhesion of cells, since protein interactions are governed by the hydrophilic/hydrophobic properties of the biological substrates. In this context, due to its tunable wettability, nHAp has been widely used as a substrate for effectively controlling surface wettability, protein interactions leading to cell adhesion and consequently bioactivity properties [32,33].

Several papers have been published using polyesters with different loads of nHAp for bone regeneration purporses [19,34,35]. Recently, Neto et al. performed an in vitro and in vivo evaluation of electrospun PBAT with different HAp loads. However, these authors evaluated only in vitro osteocalcin and a sub-cutaneous in vivo model [19].

Nevertheless, an association between in vitro assays exploring specific genes related to bone regeneration and an in vivo assay evaluating bone regeneration using electrospun PBAT with different loads of nHAp has not been systematically explored yet. For this reason, herein for the first time, a careful in vitro evaluation exploring specific genes related to bone regeneration and in vivo experiments assessed by micro-computed tomography (Micro$\mathrm{CT}$ ) and three point flexural tests were proposed to analyze bone neoformation using electrospun PBAT/nHAp composites. We performed a careful investigation examining thoroughly the influence of nHAp on the final in vitroproperties of the scaffolds, including wettability, bioactivity, gene expression of osteoblast-like MG-63 and further in vivo evaluation assessed by micro-computed tomography (MicroCT) to quantify bone growth and flexural tests to evaluate bone's resistance to fracture. Our results open new perspectives for the use of electrospun PBAT/nHAp composites as new implants for bone regeneration.

\section{Results and discussion}

All the materials and methods are described in the supplementary data. We observed that melting temperature increased with nHAp loading and as its concentration increased, whilst no clear influence was observed on glass transition. On the other hand, the nHAp loading decreased the degree of cristallinity, which may be attributed to a certain loss of mobility of the polymer chains, therefore preventing an ideal aligment of their segments in the crystal lattices (Fig. 1 and Table 2 described in the supplementary data). Then, we can evaluate whether nHAp acted as a nucleating agent or not. After polymer melting, a nucleating agent could reduce the barrier of free-energy for a primary nucleation, therefore decreasing the critical nucleus size. As a consequence of lowering the free enthalpy of nucleation, the crystallization induction time would also decrease and result in an increase in the nucleation rates $[19,36]$.

The electrospinning process is largely used to produce scaffolds that can act as support for cell adhesion and proliferation. For this purpose, the formation of fibers with proper morphology and distribution is required. The electrospun fibers were ultrathin $(100<$ diameters $<1000 \mathrm{~nm})$ and homogeneous (Fig. 1a-c). After nHAp loading, the fiber average diameter decreased from $480 \pm 140 \mathrm{~nm}$ (PBAT) to $147 \pm 10 \mathrm{~nm}$ (PBAT/3\% nHAp) and $139 \pm 19 \mathrm{~nm}$ (PBAT/5\%nHAp). Our group already observed a similar decrease using carbon nanotubes [37]. Nevertheless, as nHAp was loaded and its concentration increased, a few bead-like defects can be observed along the scaffold surfaces, which may be attributed to the formation of nHAp agglomerates/aggregates during the dissolution process.

In the biomedical field, the knowledge of material wettability can be a prerequisite to obtain a better understading of its potential applications. A material with high hydrophilicity is always desirable for tissue engineering applications. We identified that the nHAp changed the wettability properties of the electrospun PBAT scaffolds. From the contact angle curves, the advanced contact angle (ACA) values were $115^{\circ}, 101^{\circ}$ and $80^{\circ}$ for PBAT, PBAT $/ 3 \%$ nHAp and PBAT $/ 5 \%$ nHAp, respectively. Snapshots of the contact angles are shown at the top of the micrographs, before cell cultivation (Fig. 1a-c). The high values of ACA, mainly for PBAT, indicated the hydrophobic character of this polymeric matrix. Some authors relate surface hydrophilicity to the rate of cell spreading, osteoblast differentiation and improvement of metabolic activity [38-40].

These results show the possibility of tuning the wettability of the PBAT matrix by nHAp loading, further generating materials with enhanced hydrophilicity, as shown in Fig. 1a'-c', where MG-63 cells were able to adhere to the scaffolds within 1 day of cell culture, exhibiting cytoplasmic extensions and normal cell morphology. At 7 days of contact with the scaffolds, the adherent osteoblasts proliferated and spread, occupying $100 \%$ of the surface, as shown in Fig. 1a"-c". In these micrographs, typical char- 


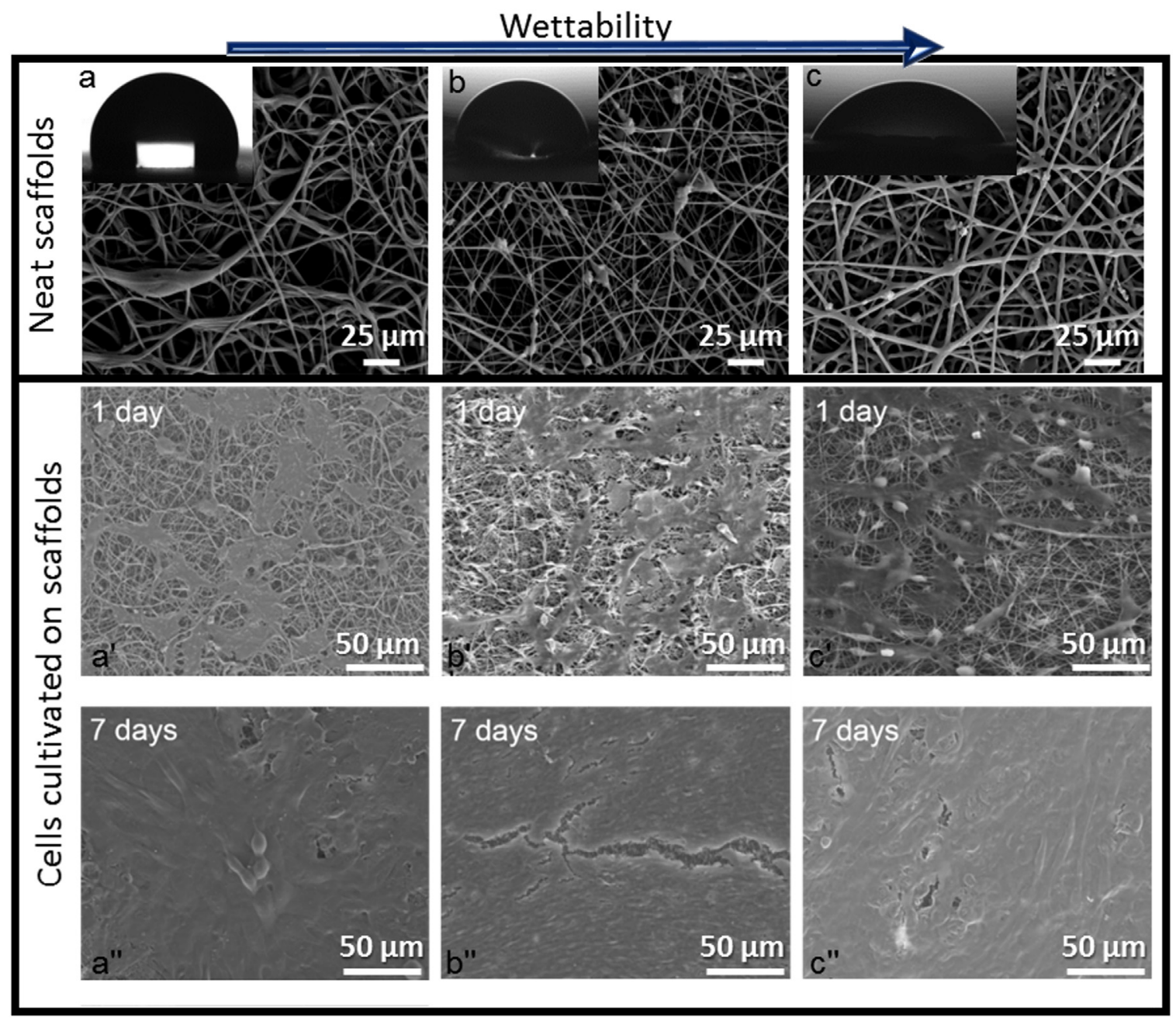

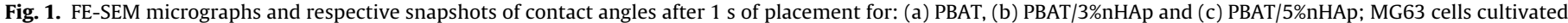

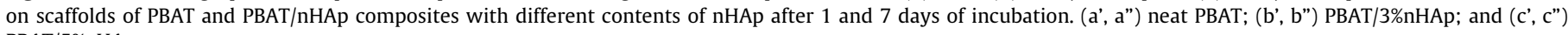
PBAT/5\%nHAp.

acteristics of cytotoxic cellular changes, such as pyknotic nuclei, fragmented cytoplasm and/or with granulation were not observed.

The best cell adhesion provides the greatest cell density which increases cell to cell contacts which positively affects osteoblast proliferation. As these cells are anchorage-dependent (meaning that they need to adhere to live), a good fixation on the scaffold surface is essential for adhesion and subsequent cell proliferation and bone formation [41]. Thus, our results indicate that the scaffolds promoted osteoblast adhesion and proliferation without evidence of cytotoxicity. As showed (Fig. 2B described in the supplementary data), the produced scaffolds increased cell viability compared to the control, corroborating our quantitative measurements of cell adhesion and proliferation (Fig. $2 \mathbf{A}$ described in the supplementary data). Similar results were obtained in studies evaluating osteoblast adhesion on PBAT scaffolds for tissue engineering [18,42].

The formation of mineralized nodules was observed by optical microscopy, which indicated the presence of calcium (Fig. 3a-d described in supplementary data) after 14 days. Higher concentrations of calcium were found in the scaffold groups in relation to the control group.Furthermore, all groups containing nHAp, independent of the concentration, showed better results compared to neat PBAT group (Fig. 3e described in the supplementary data), which corroborated their bioactive properties as reported in a previous study [43]. Staining with alizarin red is considered an important tool in osteogenesis because it is an indicator of calcium produc- tion by mature osteoblastic cells which subsequently mineralize the extracellular matrix, showing the in vitro formation of nodules [44]. However, this result alone is not sufficient to infer that the produced scaffolds are bioactive and then responsible for improving the osteogenesis process. For this, herein the gene expression was evaluated. The expression of genes and proteins involved in osteogenesis are important parameters to demonstrate an in vitro osteoblast phenotype [45]. In vitro bone formation involves the initial synthesis of a collagen matrix, subsequently mineralized by proteins secreted by mature osteoblasts $[46,47]$.

For the analysis of gene expression in this study, genes involved in osteogenesis were selected to provide perspective of this material as a scaffold for bone regeneration. All groups were subjected to quantitative PCR assay in real-time (qRT-PCR) for 3 reference genes, $\beta$-actin, GAPDH and 18s. $\beta$-actin was chosen to be the reference gene because it presents greater stability during the experimental conditions and it was used to normalize all the results (Fig. 2).

Alkaline Phosphatase (ALP) is a marker of osteogenic differentiation. The increase of its expression occurs in mature osteoblasts, as we can observe at 7 days of cell culture, where all scaffolds showed an increase in ALP expression in relation to the control group ( $p<0.001$ ), indicating the maturation of MG-63 cells in this period. However, among the scaffolds, there was a significant difference between the PBAT group and the PBAT/3\% nHAp $(\mathrm{p}<0.001)$ and $\mathrm{PBAT} / 5 \% \mathrm{nHAp}(\mathrm{p}<0.05)$ groups. At 14 days, there was a decrease 
A

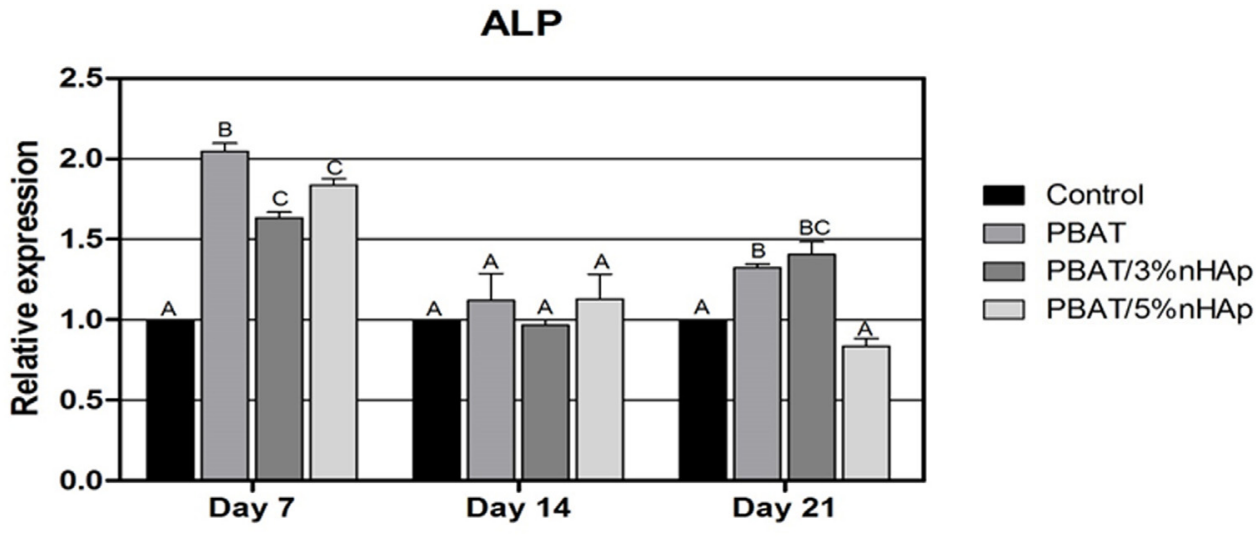

B

COL I

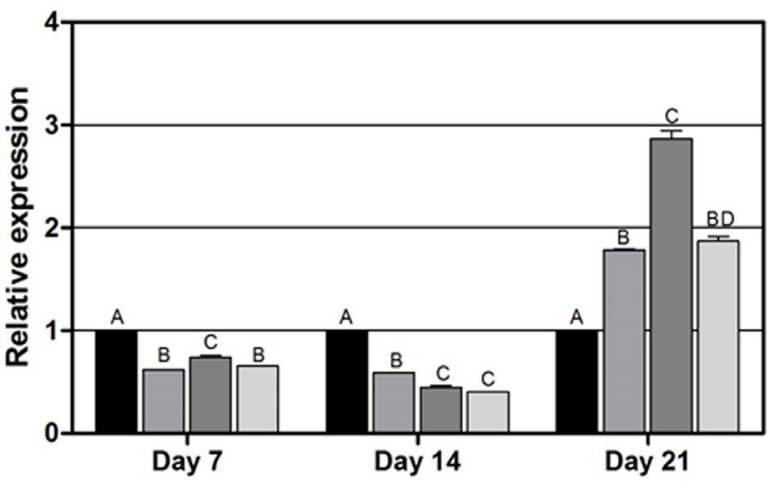

D

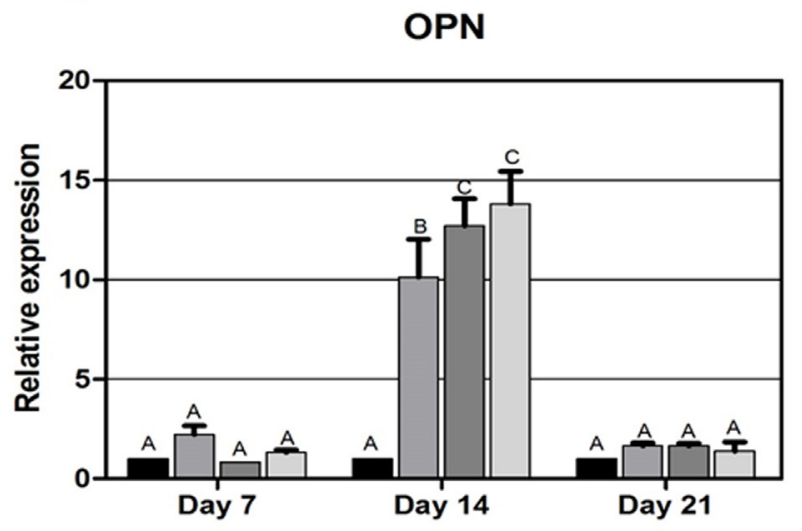

C

OC

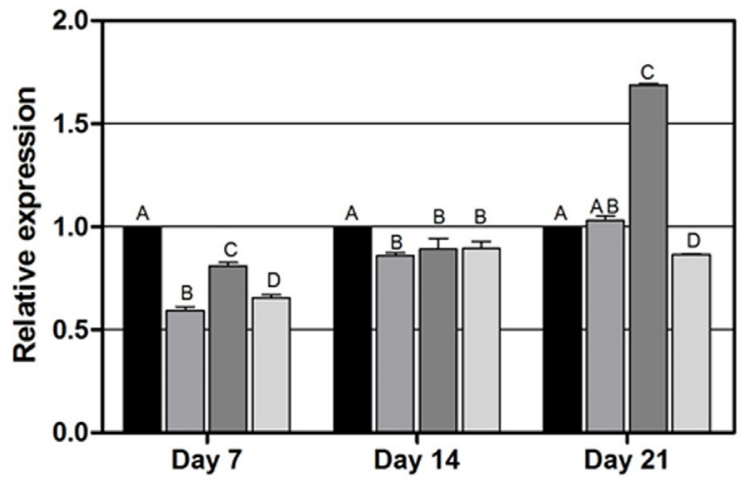

E

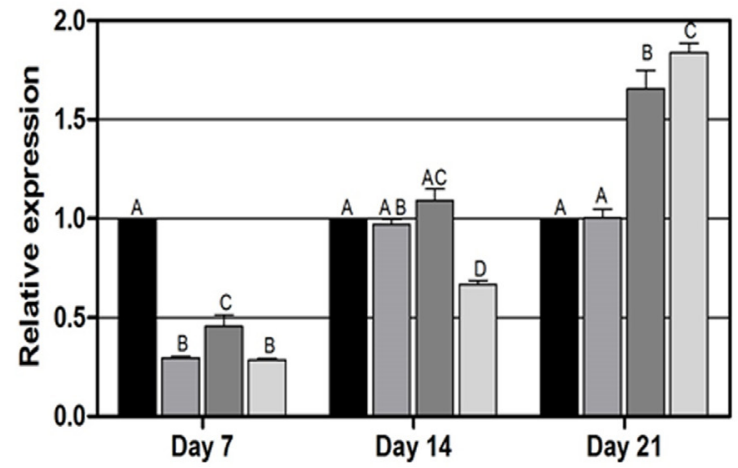

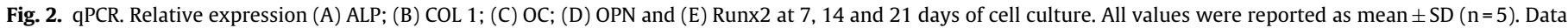
were normalized by its expression in a control well (Control $=1$ ). The letters are related to significant differences for $\mathrm{p}<0.05$. (ANOVA, post-test Tukey).

of ALP expression in the scaffolds groups, however without statistical difference between them. We identified that PBAT and PBAT/5\%nHAp groups showed higher expression of ALP than the control group. A decrease in ALP expression at day 14 was observed, probably due to the increased synthesis of mineralization proteins and the formation of a mineralized matrix as suggested by Shao et al. [48]. These data are supported by our qualitative and quantitative assays of alizarin red, with the formation of mineralized nodules observed at 14 days of cell culture. At 21 days of cell culture, there was an increase in ALP expression compared to the 14 day period, except for the PBAT/5\%nHAp group. PBAT and PBT/3\%nHAp showed no difference in ALP expression ( $p>0.05)$, but had higher expression than the control $(\mathrm{p}<0.001)$ (Fig. 2A). Similar results were obtained in previous studies in three-dimensional scaffolds, $[47,48]$ where ALP activity was higher at 7 days with subsequent reduction due to the beginning of extracellular matrix mineralization. The slight ALP increase at 21 days corroborates with Runx2 expression in the same period, and may be related to the renewal of the cell cycle [49]. 


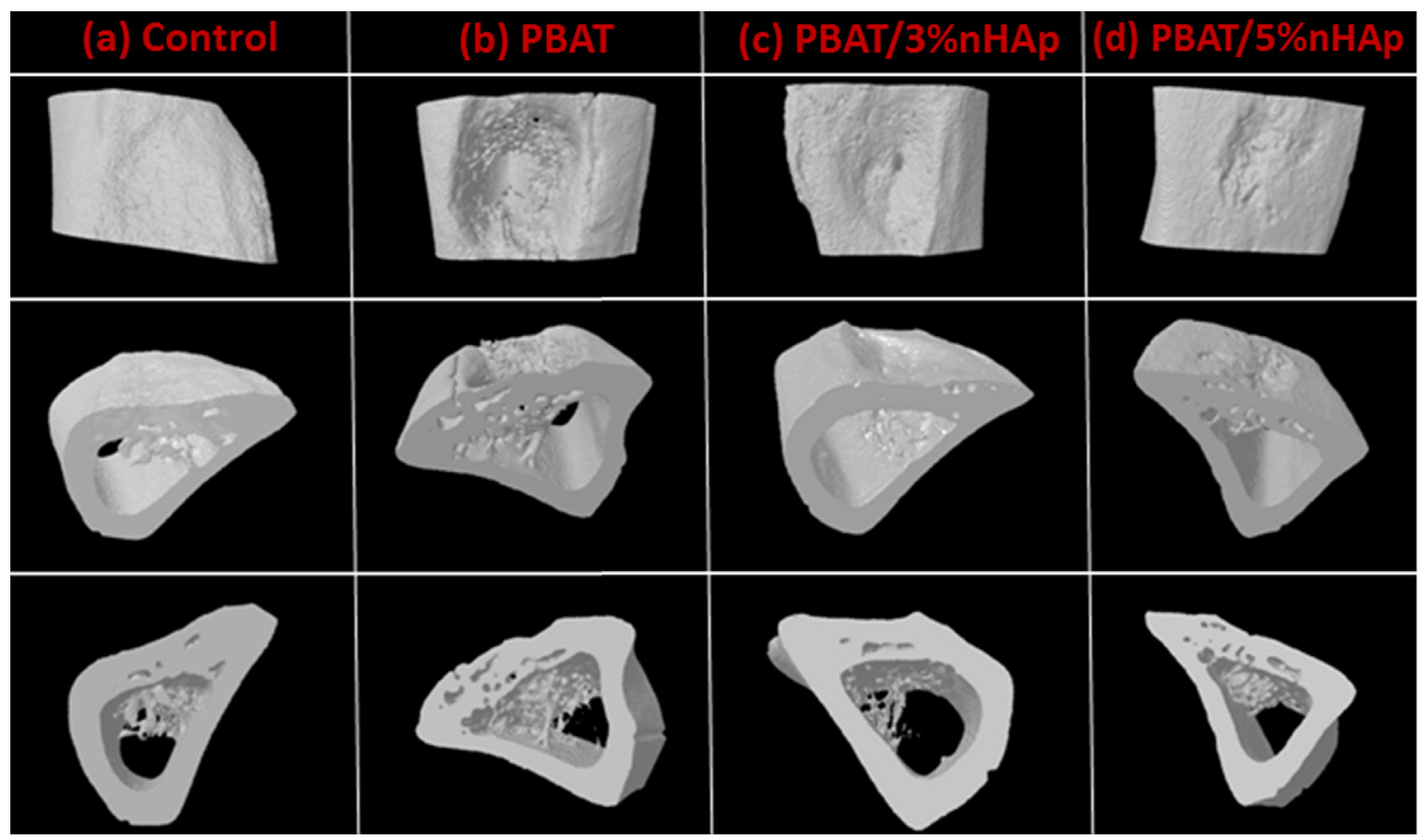

Fig. 3. Representative 3D micro-CT images of the tibia defects after 6 weeks of scaffolds implantation.
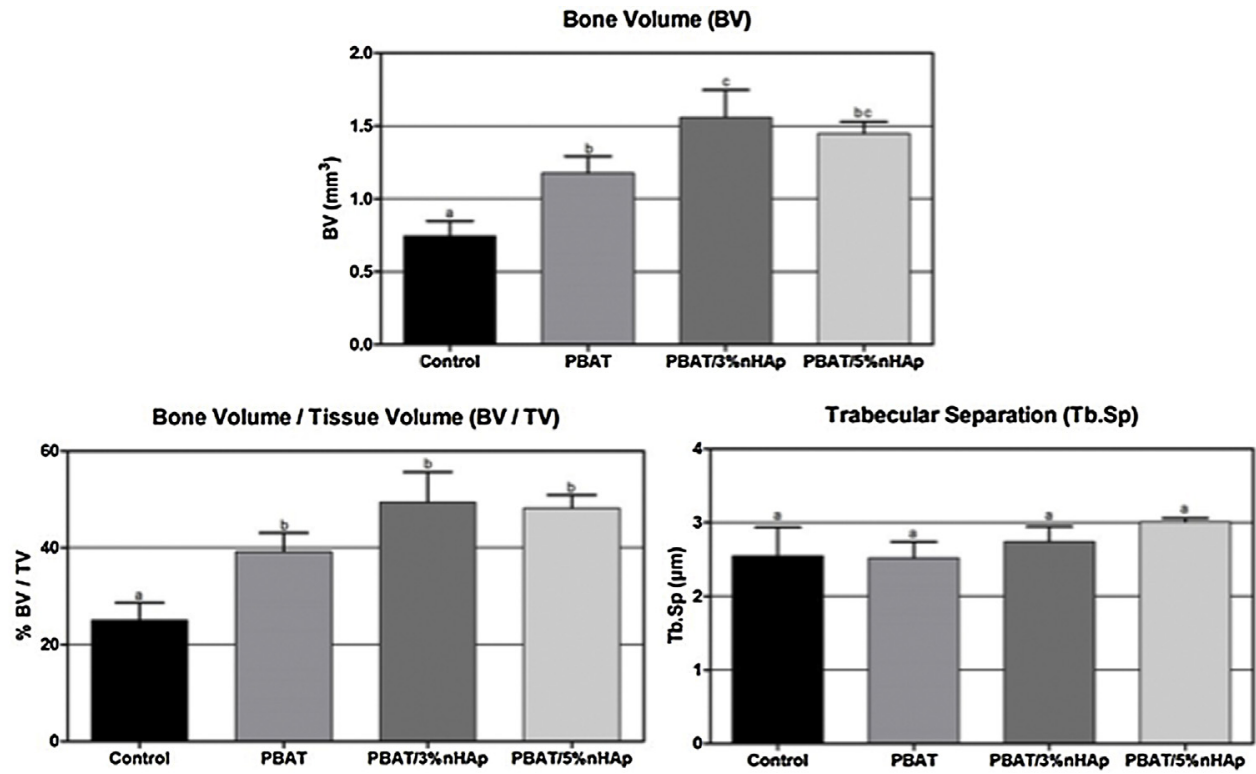

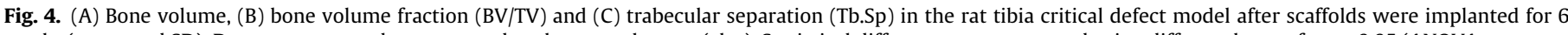

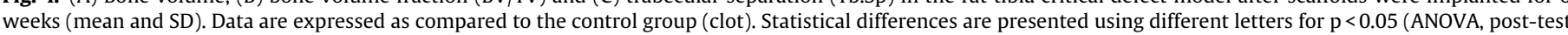
Tukey). Tb.Sp data presented no statistical difference.

The expression of Runt-related transcription factor 2 (Runx2) is associated with osteoblastic differentiation, and was decreased at 7 days of cell culture in the scaffold groups, regarding other periods and the control group $(\mathrm{p}<0.001)$. At 14 days there was an increase in scaffold gene expression, but not significant in relation to the control ( $p>0.05$ ), although there was an increase in the expression for the PBAT $/ 5 \%$ nHAp group in the period from 7 to 14 days, this group showed significantly lower expression of Runx2 compared to the other groups $(\mathrm{p}<0.001)$. However, at 21 days PBAT/3\%nHAp and PBAT/5\%nHAp showed significantly greater Runx2 expression compared to the control and PBAT groups ( $\mathrm{p}<0.001)$, and comparatively, the $\mathrm{PBAT} / 5 \% \mathrm{nHAp}$ presented higher expression than
PBAT/3\%nHAp ( $<<0.01$ ) (Fig. 2E). Runx2 expression was initially assessed at 7 days of cell culture, but Kim et al. [50] observed increased expression of Runx2 earlier, at 3 days. We suggest that the low Runx 2 expression observed in the scaffold groups at 7 days may be justified due to the mature phenotype in this period, as confirmed by high cell activity and by elevated ALP expression in the scaffold groups in the same period. Furthermore, the renewal of the cell cycle may explain the increased late expression of Runx2 at 21 days; [49] nHAp-incorporated scaffold groups had higher Runx2 expression due to the influence of nHAp in the modification of the surface wettability and then to promote osteoblastic differentiation. 

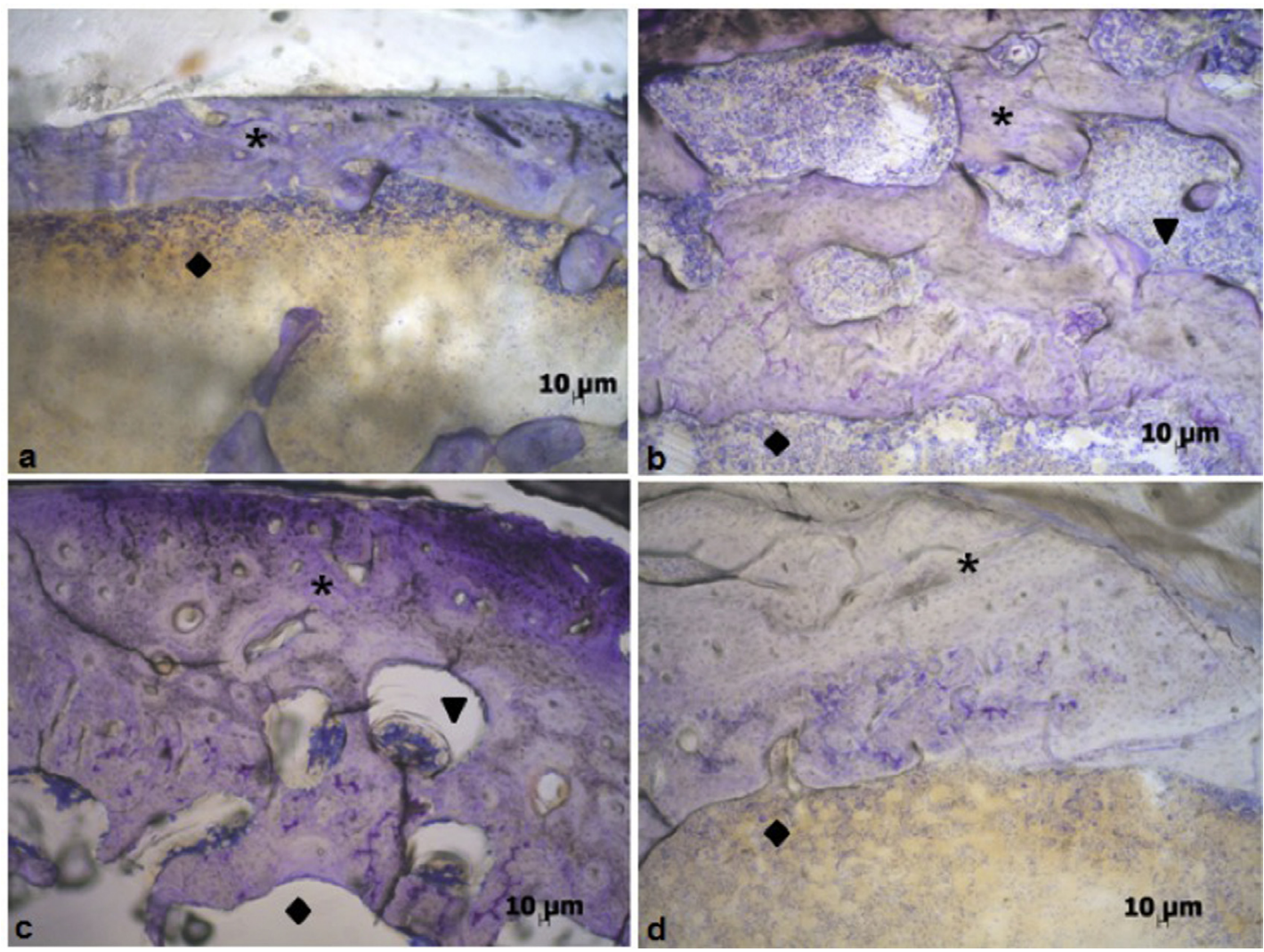

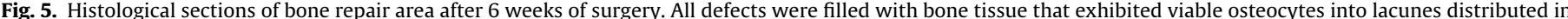

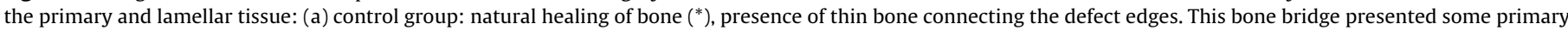

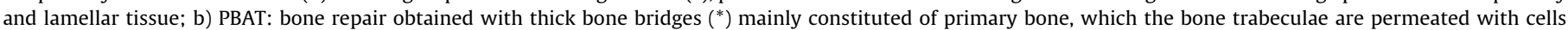

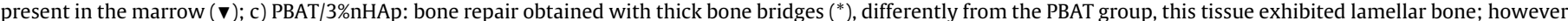

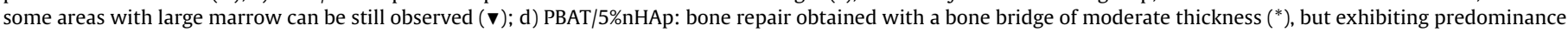
of lamellar bone. In all images, the superior side of figure shows bone formation and inferior side of figure shows the marrow space of tibia ( $)$.

Force

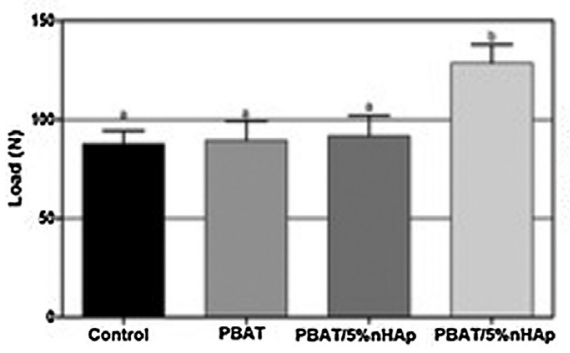

Stiffiness

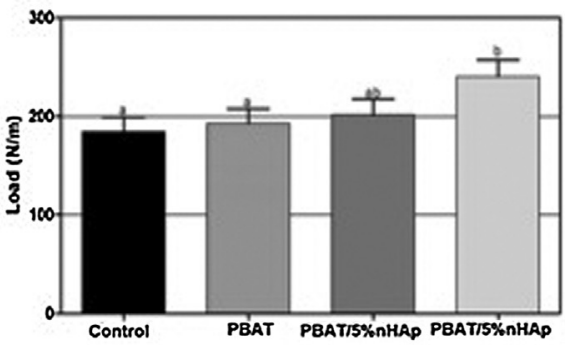

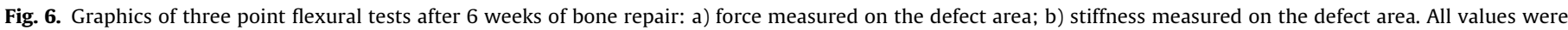
reported as mean $\pm S D(n=3)$. Different letters mean significant statistical differences for $\mathrm{p}<0.05$ (ANOVA, post-test Tukey).

The expression of Collagen type 1 (Col I), an abundant organic component of the extracellular matrix, was similar at 7 and 14 days of cell culture with higher control group expression $(\mathrm{p}>0.001)$. However, at 21 days, the scaffold groups showed a higher Col I expression than the controls ( $p<0.001)$, and, when compared, the PABT group presented similar expression to the PABT/5\%nHAp group ( $p>0.05$ ), with PABT/3\%nHAp showing the highest Col I expression compared to the others $(\mathrm{p}<0.001)$ (Fig. 2B). Although our results show less Col I expression in all scaffold groups at 7 and 14 days, periods in which the authors Ravichandran et al. [51] and Xia et al. [47] obtained an increase in Col I expression respectively, their three-dimensional architecture played a key role in the mineralized matrix formation at 14 days as found in the alizarin red assay. Clearly, an increase of Col I expression after 21 days occurred.
It was very interesting to confirm that our produced scaffolds were able to organize and calcificate the extracellular matrix.

The genes osteopontin (OPN) and osteocalcin (OC) are bone mineralization markers [52,53]. OPN expression at 7 and 21 days of cell culture was not significantly different among groups ( $p>0.05$ ), however, the scaffold groups exhibited higher OPN expression than the control group in both periods (Fig. 2D). At 14 days, statistically significant increases in OPN expression occurred for PBAT, PBAT $/ 3 \%$ nHAp and PBAT/5\%nHAp scaffolds, compared to the control group $(\mathrm{p}<0.001)$. PBAT/3\%nHAp $(\mathrm{p}<0.05)$ and PBAT/5\%nHAp $(\mathrm{p}<0.01)$ scaffolds exhibited increased expression regarding the PBAT. However, PBAT $/ 3 \%$ nHAp and PBAT $/ 5 \%$ nHAp scaffolds showed no difference in OPN expression $(\mathrm{p}>0.05)$ at 14 days.

OC expression at 7 and 14 days of cell culture in the scaffold groups significantly decreased in relation to the control group 
( $\mathrm{p}<0.001)$. However, at 7 days, the PBAT/3\%nHAp group exhibited increased expression in relation to the PBAT $(\mathrm{p}<0.001)$ and the PBAT $/ 5 \%$ nHAp $(p<0.001)$ groups, while at 14 days, there was no significant difference among scaffold groups ( $p>0.05$ ). At 21 days, there was a statistically significant increase in $O C$ expression in the PBAT $/ 3 \%$ nHAp group in relation to the other groups $(p<0.001)$ (Fig. 2C).

The high OPN expression at 14 days, as well as its distribution among the groups, corroborates our findings of mineralized nodules, a period that we evaluated and found in vitro mineralization. Alves et al. [49] reported decreased expression of some genes at 14 days and correlated it with apoptosis during the cell cycle. The constant expression of OC over time is important to support the mineralization process, and similar results were observed [54,55] where OC expression was maintained since the matrix mineralization was initiated with the consequent formation of mineralization nodules.

Briefly, the in vivo results corroborated the in vitro results. PBAT/3\%nHAp and PBAT/5\%nHAp scaffolds promoted better bone repair compared to PBAT and the control when analyzed by microCT (Figs. 3 and 4), histological analysis (Fig. 5) and flexural tests (Fig. 6). Fig. 3 shows typical 3D micro-CT images after 6 weeks of implantation. Clearly, the PBAT/nHAp groups (Fig. $3 \mathbf{c}$ and $\mathbf{d}$ (lateral view)) promoted the formation of new bone on the preexistent one, while only a depression of bone was observed using control and PBAT groups. In summary, when nHAp was incorporated, similar bone repair occurred among the groups analyzed by micro-CT (Fig. 3) and histological analysis (Fig. 5). Independently of the content used, the nHAp stimulated an increase of bone volume (Fig. 4).

Microstructural parameters of bone repair are shown in Fig. 4. PBAT $/ 3 \%$ nHAp and PBAT $/ 5 \%$ nHAp were more effective to improve bone formation, presenting higher values of BV and BV/TV compared to controls (Fig. $4 \mathbf{a}$ and $\mathbf{b})(p<0.0001)$. Then, we confirmed the effectiveness of these scaffolds to promote guided bone regeneration. Conversely, trabecular separation (Tb.Sp) was similar among all groups (Fig. 4c). We have already observed this trend in a previous work, where [56] PDLLA/nHAp scaffolds promoted better in vitro osteogenic results and in vivo bone regeneration. However, herein we performed a deeper study involving bone formation using micro-CT and mechanical properties after scaffold implantation.

The histological analyses showed the presence of bone defect in all groups (without neoformation) (Fig. 5). The control group showed one slim bridge between the edges of defect (Fig. $5 \mathbf{a}$ ) compared to other groups. PBAT/3\%nHAp (Fig. 5c) and PBAT/5\%nHAp (Fig. 5d) showed one thicker bridge than the ones for controls, indicating bone trabeculae formation. Then, the inclusion of 3 and $5 \%$ of nHAp influenced positively osseoconduction and consequently bone proliferation. Contributing for this, the BV parameter of micro-CT analysis showed a difference between control and scaffold groups. We already had observed that the association of PBAT and nHAp guided bone cell growth, independently of concentration. Also, the flexural tests confirmed previous results because when nHAp was loaded, the mechanical properties of the neoformed bone improved. (Fig. 6).

Then, the previous results corroborate the results of BV (analyzed by microCT), since higher BV values were found for the scaffolds compared to control group. We can assume that the PBAT/5\%nHAp group presented better biomechanical results compared to allbecause the predominance of lamellar bone promoted an increase of tension force at this area.

Different concentrations of nHAp associated to PBAT facilated bone neoformation. Clearly, the flexural tests for PBAT/5\%nHAp group showed better bone formation in the defective area ( $(p<0.05)$, due to high values) than all. Then, we can confirm the correlation between in vitro mineralization and in vivo bone neo- formation with superior values of mechanical properties compared to controls. Probably, the crystallite size of nHAp influenced directly bone growth due to its chemical attraction of phosphate-calcium crystals. On the other hand the stiffiness test (Fig. 6b) was not significantly different among the groups $(\mathrm{p}<0.05)$.

Although HAp is a main skeleton constituent, the mechanism involved in creating a mineralized matrix is still not fully understood, and can be more explored and explained [57]. The effectiveness of PBAT/3\%nHAp and PBAT/5\%nHAp as bone graft may be probably associated to a lower crystallinity compared to PBAT. This factor may have been responsible, respectively, for faster in vivo degradation and for enhancing bone mimetic properties that arose from the highest bioceramic content. Also, other factors may have influenced the contribution of nHAp towards improving in vivo bone growth, such as the greater wettability of the scaffold, which possibly allowed for the better interaction of proteins and changes in the phenotypic expression of osteoblasts.

Herein, for the first time we performed a systematic biological in vitro and in vivo study envolving PBAT and PBAT/nHAp scaffolds. Polyesters have been applied as scaffolds for tissue engineering. However, PBAT has an equilibrium between biodegradability and physico-chemical properties and these properties improve its flexibiliby and elongation at break. Clearly, PBAT with and without nHAp regulated in vitro osteogenesisand improved cell adhesion and proliferation. When nHAp was incorporated, the scaffolds showed bioactivity properties as already reported in previous studies $[44,58]$.

\section{Conclusions}

Herein, we performed a full in vitro and in vivo study involving $\mathrm{PBAT} / \mathrm{nHAp}$ scaffolds for bone tissue engineering. Clearly, the produced scaffolds presented in vitro biocompatibility. The attractive properties of nHAp (biomimetic structures, increased wettability, and high surface area) positively influenced in vitro osteoblast adhesion and proliferation, the formation of a mineralized matrix, as well as the expression of genes related to osteoblast differentiation and mineralization. Osteoconductive and osteoinductive effects arose from nHAp inducing better cellular responses towards scaffolds composed of PBAT/nHAp compared to neat PBAT scaffolds as well as led to more effective bone formation (confirmed by micro-CT images), which promoted the highest bone volume after 6 weeks of in vivo implantation using a well established rat tibia defect model when compared to PBAT and control group. The use of a high concentration of nHAp was important to increase the force of the neoformed bone. This investigation, thus, enhanced our comprehension on the role that nHAp may play in PBAT matrices for improving bone tissue engineering applications.

\section{Statement of significance}

Combining polyester scaffolds with synthetic nanohydroxyapatite (nHAp) is a plausible strategy to improve bone regeneration. PBAT [poly (butylene adipate-co-terephthalate)] is a very novel and interesting polyester due to its high flexibility and elongation-atbreak besides low crystallinity. Electrospinning has been applied to produce 3D fibrous scaffolds with the ability of mimicking the architecture of the bone extracellular matrix. Nevertheless, an association between in vitro assays exploring specific genes related to bone regeneration and an in vivo assay evaluating bone regeneration using electrospun PBAT with different loads of nHAp has not been systematically explored yet. Herein, we explored the specific genes related to bone regeneration and in vivo experiments by micro-computed tomography to analyze bone neoformation using electrospun PBAT/nHAp composites. 


\section{Acknowledgments}

The authors would like to thank the São Paulo Research Foundation (FAPESP, grants 2011/17877-7, 2011/20345-7, 2015/09697-0, 2016/00575-1), the National Council for Scientific and Technological Development (CNPq, 474090/2013-2), the Brazilian Innovation Agency (FINEP - grant 0113042800), the Coordination for the Improvement of Higher Education Personnel (CAPES, grant 88887.095044/2015-00) and a CAPES scholarship. B.V. M. R would also like to thank FAPESP for a postdoctoral scholarship (FAPESP, 2015/08523-8). The authors would also you like to thank Professor Ali Khademhosseini for discussions and comments.

\section{Appendix A. Supplementary data}

Supplementary data associated with this article can be found, in the online version, at http://dx.doi.org/10.1016/j.colsurfb.2017.04. 053.

\section{References}

[1] H. Hibi, Clinical review of bone regenerative medicine and maxillomandibular reconstruction, Oral Sci. Int. 13 (2016) 15-19.

[2] U. Kneser, D.J. Schaefer, E. Polykandriotis, R.E. Horch, Tissue engineering of bone: the reconstructive surgeon's point of view, J. Cell. Mol. Med. 10 (2006) 7-19.

[3] M.J. Yaszemski, R.G. Payne, W.C. Hayes, R. Langer, A.G. Mikos, Evolution of bone transplantation: molecular, cellular and tissue strategies to engineer human bone, Biomaterials 17 (1996) 175-185.

[4] G.C. Ingavle, J.K. Leach, Advancements in electrospinning of polymeric nanofibrous scaffolds for tissue engineering, Tissue Eng. Part B: Rev. 20 (2013) 277-293.

[5] G.C. Ingavle, J.K. Leach, Advancements in electrospinning of polymeric nanofibrous scaffolds for tissue engineering, Tissue Eng. Part B: Rev. 20 (2014) 277-293.

[6] L.J. Villarreal-Gómez, J.M. Cornejo-Bravo, R. Vera-Graziano, D. Grande, Electrospinning as a powerful technique for biomedical applications: a critically selected survey. J. Biomater. Sci. Polym. Ed. 27 (2016) 157-176.

[7] N. Bhardwaj, S.C. Kundu, Electrospinning: a fascinating fiber fabrication technique, Biotechnol. Adv. 28 (2010) 325-347.

[8] C.J. Angammana, S.H. Jayaram, Fundamentals of electrospinning and processing technologies, Part. Sci. Technol (2016) 1-11.

[9] T.J. Sill, H.A. von Recum, Electrospinning: applications in drug delivery and tissue engineering, Biomaterials 29 (2008) 1989-2006

[10] D. Kai, S.S. Liow, X.J. Loh, Biodegradable polymers for electrospinning: towards biomedical applications, Mater. Sci. Eng. C 45 (2014) 659-670.

[11] L. Siqueira, F.R. Passador, M.M. Costa, A.O. Lobo, E. Sousa, Influence of the addition of $\beta$-TCP on the morphology, thermal properties and cell viability of poly (lactic acid) fibers obtained by electrospinning, Mater. Sci. Eng. C 52 (2015) 135-143.

[12] S. Wu, J. Wu, J.H. Yue, M.K.T. To, H.B. Pan, W.W. Lu, X.L. Zhao, Poly (d,l-lactic acid) electrospun fibers with tunable surface nanotopography for modulating drug release profiles, Mater. Lett. 161 (2015) 716-719.

[13] F.A. Paskiabi, E. Mirzaei, A. Amani, M.A. Shokrgozar, R. Saber, R. Faridi-Majidi, Optimizing parameters on alignment of PCL/PGA nanofibrous scaffold: an artificial neural networks approach, Int. J. Biol. Macromol. 81 (2015) 1089-1097.

[14] S.B. MacKintosh, L.P. Serino, P.D. Iddon, R. Brown, R.S. Conlan, C.J. Wright, T.G. Maffeis, M.J. Raxworthy, I.M. Sheldon, A three-dimensional model of primary bovine endometrium using an electrospun scaffold, Biofabrication 7 (2015) 025010 .

[15] M. Nune, U.M. Krishnan, S. Sethuraman, PLGA nanofibers blended with designer self-assembling peptides for peripheral neural regeneration, Mater. Sci. Eng. C 62 (2016) 329-337.

[16] A.R.K. Sasikala, A.R. Unnithan, Y.H. Yun, C.H. Park, C.S. Kim, An implantable smart magnetic nanofiber device for endoscopic hyperthermia treatment an tumor-triggered controlled drug release, Acta Biomater. 31 (2016) 122-133.

[17] N. Dehdilani, K. Shamsasenjan, A. Movassaghpour, P. Akbarzadehlaleh, B.A. Tabrizi, H. Parsa, F. Sabagi, Improved survival and hematopoietic differentiation of murine embryonic stem cells on electrospun polycaprolactone nanofiber, Cell J. 17 (2016) 629-638.

[18] B.V.M. Rodrigues, A.S. Silva, G.F.S. Melo, L.M.R. Vasconscellos, F.R. Marciano, A.O. Lobo, Influence of low contents of superhydrophilic MWCNT on the properties and cell viability of electrospun poly (butylene adipate-co-terephthalate) fibers, Mater. Sci. Eng.: C 59 (2016) 782-791.

[19] W.A. Ribeiro Neto, A.C.C. De Paula, T.M.M. Martins, A.M. Goes, L. Averous, G. Schlatter, R.E. Suman Bretas, Poly (butylene adipate-co-terephthalate)/hydroxyapatite composite structures for bone tissue recovery, Polym. Degrad. Stab. 120 (2015) 61-69.
[20] L. Jiang, M.P. Wolcott, J. Zhang, Study of biodegradable polylactide/poly(butylene adipate-co-terephthalate) blends, Biomacromolecules 7 (2006) 199-207.

[21] K. Fukushima, A. Rasyida, M.-C. Yang, Characterization, degradation and biocompatibility of PBAT based nanocomposites, Appl. Clay Sci. 80-81 (2013) 291-298.

[22] R. Sepahvand, M. Adeli, B. Astinchap, R. Kabiri, New nanocomposites containing metal nanoparticles, carbon nanotube and polymer, J. Nanopart. Res. 10 (2008) 1309-1318.

[23] F. Hussain, M. Hojjati, M. Okamoto, R.E. Gorga, Review article: polymer-matrix nanocomposites, processing, manufacturing, and application: an overview, J. Compos. Mater. 40 (2006) 1511-1575.

[24] Y. Zare, I. Shabani, Polymer/metal nanocomposites for biomedical applications, Mater. Sci. Eng. C 60 (2016) 195-203.

[25] J. Venkatesan, S.K. Kim, Nano-hydroxyapatite composite biomaterials for bone tissue engineering - A review, J. Biomed. Nanotechnol. 10 (2014) 3124-3140.

[26] H. Zanin, C.M.R. Rosa, N. Eliaz, P.W. May, F.R. Marciano, A.O. Lobo, Assisted deposition of nano-hydroxyapatite onto exfoliated carbon nanotube oxide scaffolds, Nanoscale 7 (2015) 10218-10232.

[27] A. Santarelli, M. Mascitti, G. Orsini, L. Memè, R. Rocchetti, P. Tiriduzzi, F. Sampalmieri, A. Putignano, M. Procaccini, L. Lo Muzio, F. Bambini, Osteopontin, osteocalcin and OB-cadherin expression in synthetic nanohydroxyapatite vs bovine hydroxyapatite cultured osteoblastic-like cells, J. Biol. Regul. Homeost. Agents 28 (2014) 523-529.

[28] C.L. Salgado, L. Grenho, M.H. Fernandes, B.J. Colaço, F.J. Monteiro, Biodegradation, biocompatibility, and osteoconduction evaluation of collagen-nanohydroxyapatite cryogels for bone tissue regeneration, J. Biomed. Mater. Res. Part A 104 (2016) 57-70.

[29] R. Shao, R. Quan, L. Zhang, X. Wei, D. Yang, S. Xie, Porous hydroxyapatite bioceramics in bone tissue engineering: current uses and perspectives, Nippon Seramikkusu Kyokai Gakujutsu Ronbunshi/J. Ceram. Soc. Jpn. 123 (2015) 17-20.

[30] J.H. Shepherd, R.J. Friederichs, S.M. Best, Synthetic hydroxyapatite for tissue engineering applications, in: Hydroxyapatite (HAp) for Biomedical Applications, 2015, pp. 235-267.

[31] W. Suchanek, M. Yoshimura, Processing and properties of hydroxyapatite-based biomaterials for use as hard tissue replacement implants, J. Mater. Res. 13 (1998) 94-117.

[32] D. Aronov, R. Rosen, E.Z. Ron, G. Rosenman, Tunable hydroxyapatite wettability: effect on adhesion of biological molecules, Process Biochem. 41 (2006) 2367-2372.

[33] D. Aronov, G. Rosenman, A. Karlov, A. Shashkin, Wettability patterning of hydroxyapatite nanobioceramics induced by surface potential modification, Appl. Phys. Lett. 88 (2006) 163902.

[34] N.K. Nga, T.T. Hoai, P.H. Viet, Biomimetic scaffolds based on hydroxyapatite nanorod/poly(D,L) lactic acid with their corresponding apatite-forming capability and biocompatibility for bone-tissue engineering, Colloids Surf. B Biointerfaces 128 (2015) 506-514.

[35] J.-P. Chen, Y.-S. Chang, Preparation and characterization of composite nanofibers of polycaprolactone and nanohydroxyapatite for osteogenic differentiation of mesenchymal stem cells, Colloids Surf. B Biointerfaces 86 (2011) 169-175.

[36] B. Wunderlich, CHAPTER V. - the nucleation step, in: Macromolecular Physics, Academic Press, 1976, 2017, pp. 1-114.

[37] B.V. Rodrigues, A.S. Silva, G.F. Melo, L.M. Vasconscellos, F.R. Marciano, A.O. Lobo, Influence of low contents of superhydrophilic MWCNT on the properties and cell viability of electrospun poly (butylene adipate-co-terephthalate) fibers, Mater. Sci. Eng.: C 59 (2016) 782-791.

[38] W. Jianhua, I. Toshio, O. Naoto, I. Takayasu, M. Takashi, L. Baolin, Y. Masao, Influence of surface wettability on competitive protein adsorption and initial attachment of osteoblasts, Biomed. Mater. 4 (2009) 045002.

[39] D.Y. Eda, B. Robyn, P. Daphne, A. Fred, G. Selçuk, S. Wei, Accelerated differentiation of osteoblast cells on polycaprolactone scaffolds driven by a combined effect of protein coating and plasma modification, Biofabrication 2 (2010) 014109

[40] M.T. Khorasani, H. Mirzadeh, S. Irani, Plasma surface modification of poly (1-lactic acid) and poly (lactic-co-glycolic acid) films for improvement of nerve cells adhesion, Radiat. Phys. Chem. 77 (2008) 280-287.

[41] A. Przekora, K. Palka, G. Ginalska, Biomedical potential of chitosan/HA and chitosan/beta-1,3-glucan/HA biomaterials as scaffolds for bone regeneration-A comparative study, Mater. Sci. Eng. C Mater. Biol. Appl. 58 (2016) 891-899.

[42] J.G. Castro, B.V.M. Rodrigues, R. Ricci, M.M. Costa, A.F.C. Ribeiro, F.R. Marciano, A.O. Lobo, Designing a novel nanocomposite for bone tissue engineering using electrospun conductive PBAT/polypyrrole as a scaffold to direct nanohydroxyapatite electrodeposition, RSC Adv. 6 (2016) 32615-32623.

[43] J.R. Venugopal, V.R. Giri Dev, T. Senthilram, D. Sathiskumar, D. Gupta, S. Ramakrishna, Osteoblast mineralization with composite nanofibrous substrate for bone tissue regeneration, Cell Biol. Int. 35 (2011) 73-80.

[44] A. Haider, K.C. Gupta, I.-K. Kang, Morphological effects of HA on the cell compatibility of electrospun HA/PLGA composite nanofiber scaffolds, BioMed Res. Int. 2014 (2014) 11.

[45] G.R. Beck Jr., Inorganic phosphate as a signaling molecule in osteoblast differentiation, J. Cell. Biochem. 90 (2003) 234-243.

[46] N.C. Ho, L. Jia, C.C. Driscoll, E.M. Gutter, C.A. Francomano, A skeletal gene database, J. Bone Miner. Res. 15 (2000) 2095-2122. 
[47] Y. Xia, S.S. Peng, L.Z. Xie, X.J. Lian, X.J. Zhang, H. Cui, T.X. Song, F.M. Zhang, N. $\mathrm{Gu}$, F.Z. Cui, A novel combination of nano-scaffolds with micro-scaffolds to mimic extracellularmatrices improve osteogenesis, J. Biomater. Appl. 29 (2013) 59-71.

[48] W. Shao, J. He, F. Sang, B. Ding, L. Chen, S. Cui, K. Li, Q. Han, W. Tan, Coaxial electrospun aligned tussah silk fibroin nanostructured fiber scaffolds embedded with hydroxyapatite?tussah silk fibroin nanoparticles for bone tissue engineering, Mater. Sci. Eng. C 58 (2016) 342-351.

[49] L.B. Alves, V.C. Mariguela, M.F.d.M. Grisi, S.L.S. de Souza, A.B. Novaes, M. Taba, P.T. de Oliveira, D.B. Palioto, Expression of osteoblastic phenotype in periodontal ligament fibroblasts cultured in three-dimensional collagen gel, J. Appl. Oral Sci. 23 (2015) 206-214.

[50] M.J. Kim, C.W. Kim, Y.J. Lim, S.J. Heo, Microrough titanium surface affects biologic response in MG-63 osteoblast-like cells, J. Biomed. Mater. Res. A 79 (2006) 1023-1032.

[51] R. Ravichandran, S. Gandhi, D. Sundaramurthi, S. Sethuraman, U.M. Krishnan, Hierarchical mesoporous silica nanofibers as multifunctional scaffolds for bone tissue regeneration, J. Biomater. Sci. Polym. Ed. 24 (2013) 1988-2005.

[52] G.S. Stein, J.B. Lian, Molecular mechanisms mediating proliferation/differentiation interrelationships during progressive development of the osteoblast phenotype, Endocr. Rev. 14 (1993) 424-442.
[53] R.F. do Prado, F.S, de Oliveira, R.D. Nascimento, L.M.R de Vasconcellos, Y.R. Carvalho, C.A.A. Cairo, Osteoblast response to porous titanium and biomimetic surface: in vitro analysis, Mater. Sci. Eng. C-Mater. Biol. Appl. 52 (2015) 194-203.

[54] J.Y. Choi, B.H. Lee, K.B. Song, R.W. Park, I.S. Kim, K.Y. Sohn, J.S. Jo, H.M. Ryoo, Expression patterns of bone-related proteins during osteoblastic differentiation in MC3T3-E1 cells, J. Cell. Biochem. 61 (1996) 609-618.

[55] M.H. Choi, W.C. Noh, J.W. Park, J.M. Lee, J.Y. Suh, Gene expression pattern during osteogenic differentiation of human periodontal ligament cells in vitro, J. Periodontal Implant Sci. 41 (2011) 167-175.

[56] A.O. Lobo, I.A. Siqueira, M.F. das Neves, F.R. Marciano, E.J. Corat, M.A. Corat In vitro and in vivo studies of a novel nanohydroxyapatite/superhydrophilic vertically aligned carbon nanotube nanocomposites, J. Mater. Sci. Mater. Med. 24 (2013) 1723-1732.

[57] S.W. Ha, H.L. Jang, K.T. Nam, G.R. Beck Jr., Nano-hydroxyapatite modulates osteoblast lineage commitment by stimulation of DNA methylation and regulation of gene expression, Biomaterials 65 (2015) 32-42.

[58] M.P. Prabhakaran, J. Venugopal, S. Ramakrishna, Electrospun nanostructured scaffolds for bone tissue engineering, Acta Biomater. 5 (2009) 2884-2893. 